\title{
Reported incidences and factors associated with percutaneous injuries and splash exposures among healthcare workers in Kahama District, Tanzania
}

ROSE M. LAISSER ${ }^{* *}$ and JOHN F. NG'HOME ${ }^{2}$

${ }^{1}$ Archibishop Antony Mayala School of Nursing, Catholic University of Health and Allied Sciences-Bugando, P O Box 1464, Mwanza, Tanzania

${ }^{2}$ Kahama District Hospital, P O Box 472, Kahama, Tanzania

\begin{abstract}
Background: Percutaneous injuries and mucocutaneous blood and other body fluids exposure are among the common hospital hazards affecting health care workers (HCWs) worldwide. These exposures pose risks of contracting infections such as Hepatitis B and C and Human Immunodeficiency viruses. This study aimed to determine the incidence and human factors associated with percutaneous injuries and splash exposures among healthcare workers in Kahama District, Tanzania.

Methods: This descriptive cross sectional study was conducted in Kahama District of north-western Tanzania and involved randomly selected healthcare workers. Structured self-administered questionnaire was used to collect data between July and October 2015.

Results: A total of $277 \mathrm{HCWs}$ participated in the study. Among them 146 (53\%) were nurses, $138(14 \%)$ auxiliary staff $36(13 \%), 32(12 \%)$ laboratory personnel and 25 (9\%) were doctors. The mean age was 37.4 years. Seventy-one percent of the participants had more than 10 years' of working experience. About $59 \%$ of participants reported incidences of percutaneous injuries and mucocutaneous blood and other fluids exposures. About $90 \%$ of participants agreed to experience the incidences several times. While $60 \%$ disagreed with availability of personal protective gears, non-reporting of the cases was noted by $26 \%$ of participants. Majority (81\%) disagreed with existence of infection prevention and control (IPC) guidelines and protocols. The main human factors associated with the percutaneous injuries and splash exposures included HCWs experience at work (71\%), long working hours (29\%), type of workplace (48\%) and inadequate use of IPC guidelines and protocols (48\%).

Conclusion: More than half of participants reported incidents of percutaneous injuries and mucocutaneous blood and other body fluids in Kahama District of Tanzania. Adherence to universal precautions, training and reduction of long working hours are necessary in order to reduce infections from percutaneous injuries and exposures.
\end{abstract}

Keywords: Percutaneous, mucocutaneous, injuries, incidence, factors, healthcare workers, Tanzania

\section{Introduction}

Percutaneous injuries (PI) and mucocutaneous blood and other body fluids exposure (ME) are among the common occupational hazards affecting health care workers (HCWs) worldwide (Shah et al., 2003; Tarantola et al., 2006). Percutaneous injuries refer to occupation-related unintentional injuries that break the integrity of the skin. The incidences of PI commonly occur during administration of injections, withdrawing blood, recapping needles, disposing of needles and handling trash (Tarantola et al. 2006). Mucocutaneous splash exposures occurs when there is contact of the body mucous membrane with blood, other body fluids, tissues, or specimens at the healthcare facility (Amira \& Awobusuri, 2014). ME happens during invasive procedures or when handling high risk fluids (CDC, 1992; WHO, 2005). Statistics indicate that 3 million percutaneous injuries occur annually among 35 million healthcare workers globally and over $90 \%$ of them happen in resource constrained countries (Prüss-Ustün et al., 2005). HCWs in Africa suffer approximately two to four percutaneous injuries per year (Gupta et al., 2008). Nigeria, Tanzania and South Africa report about 2 cases of percutaneous injuries per $10 \mathrm{HCWs}$ (WHO, 2005). A study which was conducted at Bugando Medical Centre in north-western Tanzania reported a percutaneous injury prevalence of $48.6 \%$ (Chalya et al., 2015).

\footnotetext{
*Correspondence E-mail: roselaisser.rl@gmail.com
} 
Percutaneous injury and mucocutaneous splash exposures are a substantial sources of infections with blood borne pathogens among health care workers. Worldwide, occupational exposures accounts for $2.5 \%$ of HIV cases and $40 \%$ of Hepatitis B and C cases among HCWs (PrüssUstün et al., 2005; Gupta et al., 2008). In a cross sectional study conducted at two regional hospitals in Pwani and Dodoma regions of Tanzania found that 68.8 \% of HCWs are at higher risk of HIV infection and that the estimated risk of HIV transmission due to needle stick injuries is 7 cases per 1,000,000 HCWs-years (Mashoto et al., 2013). Infection control measures and adherence to routine standard precautions are known to significantly reduce the risks of HIV and Hepatitis transmission among HCWs (Prüss-Ustün et al., 2005; Gupta et al., 2008; Hanafi et al., 2011). However, frequent incidents of PI and ME causes exposed HCWs to experience fear, anxiety and emotional distress (Jahan, 2005) and deter their motivation to comply with universal standard precautions (de la Tribonnière et al., 1998; Worthington et al., 2006; Chalya et al., 2016).

Majority of the previous studies attributed institutional and environmental factors to this situation. This study considered human factors associated with PI and ME among HCWs. Human factors include HCW individual factors such as being aware or not on effects of PI and $\mathrm{ME}$, experiences of a HCW on occurrences of the injuries and exposures, its perceived risks and factors surrounding the incidences such as workload and other practice behaviours. Therefore, the objective of this study was to determine the incidence and human factors contributing to percutaneous injury and mucocutaneous splash exposure among healthcare workers in Kahama district health facilities Tanzania.

\section{Materials and Methods}

\section{Study design and setting}

This descriptive cross-sectional study was conducted in Kahama district and included healthcare works in both private and public health facilities. Kahama district is located in Shinyanga Region, northwest of Tanzania. The district is served by 31 healthcare facilities, of which 12 are public and 19 are private owned.

\section{Study population and sampling}

The study population included all health-care workers who came into contact with patients and those who were potentially exposed to blood and other body fluids from patients. They comprised of doctors, clinical officers, nurses, laboratory personnel, mortuary attendants and housekeeping staff. The HCWs whose jobs were not directly related to health care delivery and did not handle patients' specimens were excluded from this study. A sample of size of 277 HCWs was obtained by using Kish's formula (1965) from the study population of 731 (based on district statistics of 2014).

The HCWs were recruited according to their cadres (that is doctors, nurses and others) using a stratified, simple random sampling method. In collaboration with the health facility management a list of staff per cadre was obtained. Each cadre was labelled as stratum. A simple random sampling method was then used to get participants per each stratum proportionally to reach a total of 277 participants.

\section{Data collection}

Data was collected using pre-tested structured self-administered questionnaire with questions arranged in 4 parts, A, B and C. and D. In part A, questions related to demographic characteristics of participants were included. In part $B$ issues related healthcare workers on their human factors concerning PI were asked while in part $C$ questions geared to find out information the HCWs reported about blood and body fluids exposures. The questionnaire was in a Likert scale format for parts B and C; where respondents were asked to agree or disagree with several statements related to the study. For part $D$ questions related to risk 
factors for $\mathrm{PI}$ and $\mathrm{ME}$ such as whether the respondents were aware of $\mathrm{ME}$ and about injection practices. This part also included information of infection prevention practices such as whether they used personal protective equipment during invasive procedures. The English version was constructed initially then translated into Kiswahili followed by back to back translation to ensure language proficiency and accuracy. The filled in self-administered questionnaires were reviewed for completeness and consistency by the researchers before they left the field. The questionnaire was coded, edited and data was cleaned.

\section{Data analysis}

Data was entered and analysed by SPSS software version 21.0. Measures of central tendency were used to describe the continuous variables. Chi-square test was run to test for significance of associations between the human factors and dependent variables. Significance was set at p-value less than 0.05 .

\section{Ethical considerations}

Ethical approval was sought from the joint Catholic University of Health and Allied Sciences and Bugando Medical Centre Ethics Review Committee. Permission for the study was obtained from Kahama District Authorities. Written informed consent was asked from participants' prior the study.

\section{Results}

\section{Basic characteristics of participants}

A total of $277 \mathrm{HCWs}$ consented and participated in this study. Their mean age was 37.4 years (range $=20-56$ years). More than half $(56.3 \% \mathrm{n}=156)$ were females while at least half $(55.2 \%$ $n=145$.), had secondary level of education. More than half $(55.6 \% n=154)$ had basic certificates in health related courses with $52.7 \%(n=147)$ of them being nurse midwives. Seventy-five respondents $(26.7 \%)$ were working in the surgical wards. Majority $(70.8 \% ; n=196)$ of the HCW had less than 10 years' working experience (Table 1 ).

Table 1: Socio-demographic characteristics of the participants

\begin{tabular}{llll}
\hline Variable & Response & Frequency (n) & Percentage \\
\hline Sex & Male & 121 & 43.7 \\
Education & Female & 156 & 56.3 \\
& College/University & 89 & 32.1 \\
Working department & Secondary & 153 & $55 \cdot 3$ \\
& Primary & 35 & 12.6 \\
& Emergency & 7 & 2.5 \\
& Paediatric Ward & 31 & 11.2 \\
& Maternity ward & 54 & 19.5 \\
& Laboratory & 37 & 13.4 \\
& Theatre & 15 & 5.4 \\
Working experience & Medical ward & 59 & 21.3 \\
& Surgical ward & 75 & 26.7 \\
Professional qualification & <10 years & 196 & 70.8 \\
& D10 years & 81 & 29.2 \\
& Degree & 6 & 2.2 \\
Cadre & Advanced Diploma & 17 & 6.1 \\
& Certificate & 100 & 36.1 \\
& Medical doctor & 25 & 9.0 \\
& Nurse & 146 & 52.7 \\
& Clinical officer & 36 & 13.0 \\
& Laboratory technician & 32 & 11.6 \\
& Medical attendant & 38 & 13.7 \\
\hline
\end{tabular}




\section{Incidences of percutaneous injuries and mucocutaneous blood exposure}

A total of 164 (59.2\%) participants reported to experience incidents of PI and ME in the previous oneyear prior the study. Among them $34.7 \%$ of participants encountered PI incidents while $24.5 \%$ of the participants had ME incidents. Those with more than 10 years of working experience reported more occurrences of $M E$ in the previous year than their counterparts less than 10 years of work experience (33.3. \% v/s 11. $0 \%$; $p=0.002$ ). More (48. 4\%; $p=0.004) \mathrm{HCWs}$ at the paediatric ward reported PIs incidences compared than those from other wards/departments. The HCWs who had 40 or more hours of work reported more incidences (28.9\%) of ME than those who worked for less than 40 hours per week $(14.9 \%)(p=0.001)$. Participants who knew the types of PPE available at their departments indicated reported to have experience few incidences of PI and ME in the previous year compared to those who reported not to be aware of available PPE in their departments (21\%. versus $43.5 \%$; $\rho=0.003$ ).

\section{Actions taken by health workers following PI and ME incidents}

Majority of the participants reported to wash the site with soap and running water (67.9\%) after an incident of PI while $63.8 \%$ reported washing the site with soap and running after after an incident of ME (Table 2).

Table 2: Actions taken by healthcare workers after PI or ME incident

\begin{tabular}{|c|c|c|c|}
\hline Type of injury exposure & Action taken & Number & Percentages \\
\hline \multirow[t]{4}{*}{$\begin{array}{l}\text { Percutaneous injuries }(\mathrm{N}= \\
106)\end{array}$} & $\begin{array}{l}\text { Washing the site with soap and } \\
\text { running water }\end{array}$ & 72 & 67.9 \\
\hline & Cleaning the site with antiseptic agents & 14 & 13,2 \\
\hline & Squeezing the injured site & 12 & 11.3 \\
\hline & No action taken & 8 & 7.5 \\
\hline \multirow{4}{*}{$\begin{array}{l}\text { Mucocutaneous exposures } \\
(\mathrm{N}=58)\end{array}$} & Washing the site with running water & 37 & 63.8 \\
\hline & Cleaning the site with antiseptic agents & 12 & 20.7 \\
\hline & Washing the site with normal saline & 7 & 12.1 \\
\hline & No action taken & 2 & 3.4 \\
\hline
\end{tabular}

\section{Factors associated with PI and ME}

The majority $(80.5 \%)(n=223)$ of participants strongly agreed / agreed to handle cases of PIs and ME among other staff while $89.5 \%(n=248)$ were aware of the risks of contracting hepatitis $B$ and $C$ infection. More than half $(66.4 \%) n=184)$ of the participants had personal protective equipment (PPE)in their departments. About $68.5 \%, n=190$ ) of HCWs also strongly agreed/agreed on awareness of staff safety management procedures. Majority $(80.5 \%, n=223)$ denied the existence of IPC guidelines and protocols. About $90 \%(n=249)$ of participants strongly agreed/agreed to have experienced PI or ME several times in the previous year. Over $28 \%(n=78)$ of participants strongly disagreed /disagreed that PI and ME posed risks while $25.9 \%,(n=72)$ of them disagreed with the necessity to report PI or ME. incidents. About 33\%, $(n=92)$ did not always use PPE (Table 3 ).

\section{Reasons for not reporting incidences of PI and ME}

Apart from exploring human factors also respondents were asked to mention reasons that contribute to poor reporting of $\mathrm{PI}$ and $\mathrm{ME}$ incidences in their working areas. As summarized in Table 4 , the findings revealed that $60.0 \%$ lacked knowledge of the appropriate procedures to follow after an incident (Table 3 ). 
Table 3: Perceptions of healthcare workers on PI and ME protection issues

\begin{tabular}{|c|c|c|c|c|}
\hline \multirow[t]{2}{*}{ Statement on PI/ME protection } & \multicolumn{2}{|c|}{ Strongly agree/Agree } & \multicolumn{2}{|c|}{ Strongly disagree/Disagree } \\
\hline & No. & $\%$ & No. & $\%$ \\
\hline $\begin{array}{l}\text { Percutaneous injuries occur under the skin } \\
\text { caused by sharps }\end{array}$ & 96.8 & 268 & 3.2 & 9 \\
\hline $\mathrm{PI}$ and ME are risky for my health & 71.8 & 199 & 28.2 & 78 \\
\hline $\begin{array}{l}\text { Percutaneous injuries and ME can be } \\
\text { prevented }\end{array}$ & 88.1 & 244 & 11.9 & 33 \\
\hline $\begin{array}{l}\text { Experience PI and exposures of ME several } \\
\text { times in my work }\end{array}$ & 89.8 & 249 & 10.2 & 28 \\
\hline $\begin{array}{l}\text { Likely to injure with sharps and expose to } \\
\text { splashes of blood/other body fluids when } \\
\text { engaged with many patients at a time }\end{array}$ & 33.2 & 40.1 & 20.2 & 6.5 \\
\hline $\begin{array}{l}\text { Should report immediately when encountering } \\
\text { any } \mathrm{PI} \text { or } \mathrm{ME} \text { incident }\end{array}$ & 74.0 & 205 & 25.9 & 72 \\
\hline Always put personal protective effect at work & 66.6 & 185 & 33.3 & 92 \\
\hline $\begin{array}{l}\mathrm{PI} \text { and } \mathrm{ME} \text { are more common in labour and } \\
\text { mortuary sections / rooms }\end{array}$ & 83.7 & 232 & 16.3 & 45 \\
\hline $\begin{array}{l}\text { Ignorance of risks of PI and ME may cause not } \\
\text { reporting of the incidence }\end{array}$ & 79.0 & 219 & 21.0 & 58 \\
\hline $\begin{array}{l}\text { Adherence to practice prevents the risks to } \\
\text { encounter } \mathrm{PI} \text { and } \mathrm{ME}\end{array}$ & 81.2 & 225 & 18.8. & 52 \\
\hline
\end{tabular}

Table 4: Reasons for not reporting PI and ME incidences among healthcare workers

\begin{tabular}{lll}
\hline Reasons for not reporting & Frequency & Percentages (\%) \\
\hline Lack of awareness of the appropriate procedures after injury & 90 & 60.0 \\
Thought the source was not infectious & 18 & 12.0 \\
Not knowing who to report to & 12 & 8.0 \\
Belief that their HBV vaccination status was sufficient & 9 & 6.0 \\
Time constraints & 7 & 4.7 \\
Fear of punitive response by employer & 4 & 2.7 \\
No reason given & 10 & 6.6 \\
\hline
\end{tabular}

\section{Discussion}

This study found that majority of HCWs were young adults in their mid-thirties. More than half of the HCWs comprised of nurses as reported by a similar study in a tertiary hospital in north-western Tanzania (Chalya et al., 2016). It has been reported that nurses form the majority of health work force worldwide (Squires et al., 2016). The current study also showed that almost three quarters' of $\mathrm{HCWs}$ had certificate level of professional education with fewer years of pre-service training. The fact that the certificate cadre basic training is shorter with a maximum of 2 years indicated fewer content areas on infection prevention competences and raised the need for concrete infection prevention trainings at work (Chalya et al., 2016). Over three quarters' of HCWs had more than 10 years of work experience and had experienced IP and ME exposures more than the less experienced staff. Experienced staff are anticipated to trust themselves at work and hence fail to observe new guidelines such as the current universal precautions. Contrary to our finding a study in Malaysia reported that majority of less experienced HCWs frequently encountered occupational injuries and blood exposures ( $\mathrm{Ng} \&$ Hassim, 2007).

About three quarters of the HCWs reported to have experienced several incidences of PI or $M E$. This finding concur with studies in Ethiopia (Gessessew \& Kahsu, 2006; Reda et al., 2010), Kenya (Mbaisi et al., 2013), Malaysia (Ng \& Hassim, 2007), Nigeria (Olifi et al., 2004; Ibekwe et al., 2014; Isara et al., 2015), Tanzania (Manyele et al., 2004; Chalya et al., 2015), Uganda (Nsubuga \& Jaakkola, 2005) and United Arab Emirates (Jacob et al., 2010). Despite variations in figures reported in these studies 
it indicated that many HCWs were at greater risks of contracting blood-borne infections which necessitates the need for urgent safety and protection measures in resource poor countries.

A larger proportion of HCWs from paediatric department reported incidence of $\mathrm{PI}$ and/or ME compared to others. Long working hours are attributed to physical exhaustion and stress and hence increases chances of human errors that contributed to risk behaviours among HCWs. Long hours also indicated shortages of staff which further raised emotional upsets and stress burn-outs. We reported one third of the HCW not to always use PPE in their daily activities that could have resulted from the mentioned reasons. More than half of HCWs had no PPE in their departments. Inadequate availability of PPE has been reported by other studies in East Africa (Nsubuga \& Jaakkola, 2005; Chalya et al., 2015). In this study, HCWs who were unaware of guidelines at their workplaces reported more occupational exposures compared to those who were aware of the guidelines in their work places.

Low reporting of PI and ME incidences among HCWs found in the current study was attributed by lack of infection prevention guidelines and protocols in the departments coupled with workload constraints. Similarly, the HCWs did not know whom to report to after an incidence of PI or ME. Contrary to our study other reasons for non-reporting by HCWs in a University Hospital Alexandria showed that the doctors and nurses believed that HBV was adequate (Hanafi et al, 2011). Unreported $\mathrm{PI}$ and ME posed serious problems and they prevented injured HCWs from receiving post exposure prophylaxis against HIV and other services. Findings indicated that most HCWs understood the recommended actions to be taken following a PI or ME. However, most often clean running water was not available at the health facilities (data not shown). This later observation is an indicator that human factors surrounding PI and ME were indirectly coupled with institutional and organizational factors as reported in some studies (Mbaisi et al., 2013; Chalya et al., 2016).

This study had a number of limitation, including the study design and the used selfadministered structured questionnaire. Interpretation and understanding of the questions set in the tool might slightly vary between the participants. The anticipated variation might have effected on participants' responses. Recall bias and exaggerations in responses for perception question might have also affected the results.

In conclusion, the study provided evidence on reported incidences of percutaneous injury and mucocutaneous splash exposure that occurred to healthcare workers in Kahama District of Tanzania. The human factors associated with such occurrences reported in the study meant to guide for the best measures that will ensure healthcare works are protected from contracting bloodborne infections. Since, adherence to infection prevention guidelines is known to be protective against occupational injuries, training on infection prevention strategies, continuous availability of personal protective equipment and measures to ensure reduction of long working hours are mandatory.

\section{Acknowledgements}

The authors are grateful to many people who supported the team during this study. Special thanks goes to the Management of Kahama town council Health facilities for permission to conduct this study. We also thank all participants for their time and consent to join this study.

\section{References}

Amira, C.O. \& Awobusuyi, J.O. (2014) Needle-stick injury among health care workers in hemodialysis units in Nigeria: a multi-center study. International Journal of Occupational Medicine and Environmental Health 5:1-8.

CDC (1992) Update: human immunodeficiency virus infections in health-care workers exposed to blood of infected patients. MMWR 36, 285-289. 
Chalya, G., Mbunda, F. \& Chalya, P.L. (2016) Knowledge, practice and factors associated with poor compliance with universal precautions among healthcare workers at Bugando Medical Centre, Mwanza, Tanzania. Tanzania Journal of Health Research 18 (3): Doi: http://dx.doi.org/10.4314/thrb.v18i3.3

Chalya, P.L, Seni, J., Mushi, M.F., Mirambo, M.M., Jaka, H., Rambau, P.F., Mabula, J.B., Kapesa, A., Ngallaba, S.E., Massinde, A.N. \& Kalluvya, S.E. (2015) Needle-stick injuries and splash exposures among health-care workers at a tertiary care hospital in north-western Tanzania. Tanzania Journal of Health Research 17 (2) doi: http://dx.doi.org/10.4314/thrb.v17i2.3

de la Tribonnière, X., Dufresne, M.D., Alfandari, S., Fontier, C., Sobazek, A., Valette, M., Ajana, F., Gerard, Y., Maulin, L., Bourez, J.M., Baclet, V., Senneville, E., Vermersh, A. \& Mouton Y. (1998) Tolerance, compliance and psychological consequences of post-exposure prophylaxis in health-care workers. International Journal of STD and AIDS 9: 591-594.

Gessessew, A. \& Kahsu, A. (2006) Occupational exposure of health workers to blood and body fluids in six hospitals of Tigray region: Magnitude and management. Ethiopian Medical Journal 47: 213.

Gupta, A., Shuchi, A., Jayagowri, S., Krisagar, A., Basavaraj, A., Bhat, S.M., Gupte, N., Bollinger, C.R. \& Kakrani, L.A. (2008) High risk for occupational exposure to HIV and utilization of postexposure prophylaxis in a teaching hospital in Pune, India. BMC Infectious Diseases 8:142.

Hanafi, M.I., Mohamedi, A.M., Kassem, M.S. \& Shawki, M. (2011) Needle stick injuries among health care workers of University of Alexandia Hospitals. East Mediterranean Health Journal 17: 26-35.

Ibekwe, R.N. \& Adam, V.Y. (2014) Injection safety practices among resident doctors in a tertiary health facility in Benin City. Nigeria Journal of Clinical Practice 17: 403-406.

Isara, A.R., Oguzie, K.E. \& Okpogoro, O.E (2015) Prevalence of needle stick injuries among healthcare workers in Accident and Emergency Department of a teaching Hospital in Nigeria. Annals Medical and Health Science Research 5: 392-6.

Jacob, A., Newson-Smith, M., Murphy, E., Stener, M. \& Dick, F. (2010) Sharps injuries among healthcare workers in the United Arab Emirates. Occupational Medicine 60: 395-397.

Jahan, S. (2005) Epidemiology of needles tick injuries among health care workers in a secondary care hospital in Saudi Arabia. Annals Saudi Medicine 25:233-238.

Kish, L. (1965) Survey Sampling. New York: John Wiley and Sons, Inc.

Manyele, S.V., Ngonyani, H.A. \& Eliakimu, E. (2008) The status of occupational safety among health service providers in hospitals in Tanzania. Tanzania Journal of Health Research 10: $156-165$.

Mashoto, K.O., Mubyazi, G.M., Mohamed, H. \& Malebo, H.M. (2013) Self-reported occupational exposure to HIV and factors influencing its management practice: a study of healthcare workers in Tumbi and Dodoma Hospitals, Tanzania. BMC Health Services Research 13:276.

Mbaisi, E.M, Ng'ang'a, Z., Wanzala, P. \& Omolo, J. (2013) Prevalence and factors associated with percutaneous injuries and splash exposures among health-care workers in a provincial hospital, Kenya. Pan African Medical Journal 14:10.

Ng, Y.W. \& Hassim, I.N (2007) Needle stick injury among medical personnel in Accident and Emergency department of two teaching hospitals. Medical Journal of Malaysia 62: 9-12.

Nsubuga, F.M. \& Jaakkola, S.M. (2005) Needle-stick injuries among nurses in Sub-Saharan Africa. Tropical Medicine and International Health 10: 773-781.

Ofili, A.N., Asuzu, M.C. \& Okojjie, O.H. (2003) Knowledge and practice of Universal precautions among nurses in central hospital, Benin city, Edo State Nigeria. Nigerian Postgraduate Medical Journal 10: 26-31.

Prüss-Ustün, U.A., Rapiti, E. \& Hutin, Y. (2005) Estimation of global burden of disease attributable to contaminated sharps injuries among healthcare workers. American Journal of Industrial Medicine 48: 482-490. 
Reda, A.A., Fisseha, S., Mengistie, B., \& Vandeweerd, J-M. (2010) standard precautions: occupational exposure and behavior of health care workers in Ethiopia. PloS One 5 (12): e14420.

Salelkar, S., Motghare, D.D., Kulkani, M.S. \& Vaz, F.S. (2010) Needle stick injuries among heath care workers at a tertiary care hospital. Indian Journal of Public Heath 54:18-20.

Shah, S., Bonauto, D., Silverstein B., Foley, M. (2005) Worker's compensation claims for needle stick injuries among health care workers in Washington State, 1996-2000. Infection Control Hospital Epidemiology 26: 775-781.

Squires, A., White, J., \& Simeons, W. (2016) Quantity and relevance of nursing workforce for patients' outcomes. ICN Policy Brief, Geneva, International Council of Nurses.

Tarantola, A., Abiteboul, D. \& Rachline, A. (2006) Infection risks following accidental exposure to blood or body fluid in health care workers: A review of pathogens transmitted in published cases. American Journal of Infection Control 34: 367-375.

WHO (2005) Reducing risks, promoting healthy life the world health report Geneva: World Health Organization.

Worthington, M.G., Ross, J.J. \& Bergeron, E.K. (2006) Posttraumatic stress disorder after occupational HIV exposure: Two cases and literature review. Infection Control and Hospital Epidemiology 27: 215-217.

Zafar, A., Habib, F., Hadwani, R. Ejaz, M. Kwavaja, K. Kwavaja, R. \& Irfan, S. (2009) Impact of infection control activities on the rate of needle stick injuries at a tertiary care hospital of Pakistan over a period of six years: an observational study. BMC Infectious Disease 9:78. 